ORIGINAL ARTICLE

\title{
Translation of the Amsterdam Preoperative Anxiety and Information Score (APAIS) into the Amharic Version and Its Validation for Evaluation of Preoperative Anxiety
}

\author{
Blen Ayele ${ }^{1}$, Mahelet Tadesse ${ }^{1}$, Rahel Tilahun', Berhanu Nega ${ }^{2 *}$
}

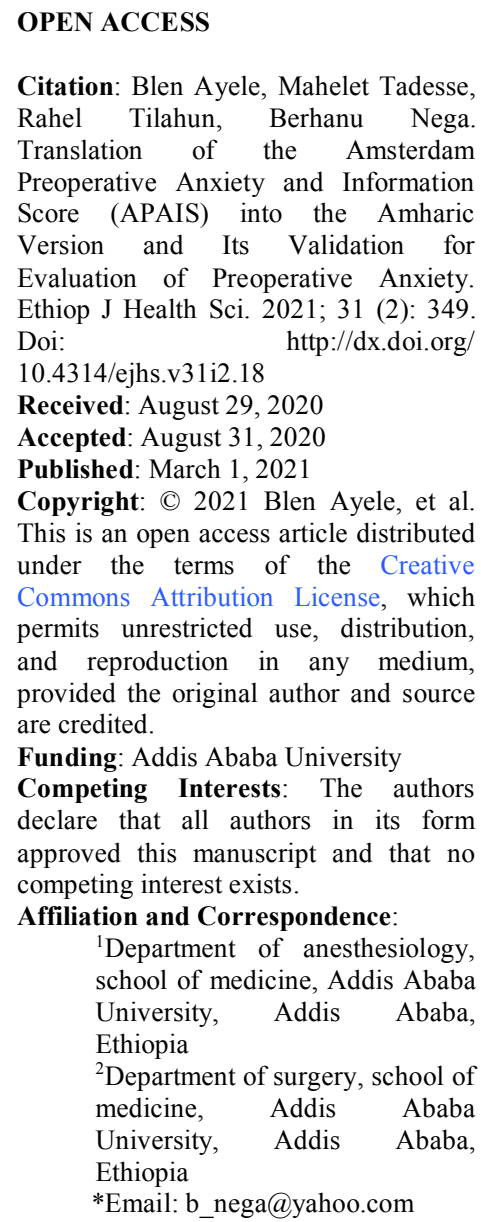

\section{ABSTRACT}

BACKGROUND: Peri-operative anxiety is a vague, unpleasant feeling, the source of which is often nonspecific and unknown to the individual. It affects all aspects of anesthesia. Although the magnitude and consequences of preoperative anxiety are well documented in the developed world, there are limited studies conducted in Ethiopia. The primary aim of this study is to produce the Amharic version of APAIS and evaluate its validity in assessing the prevalence of preoperative anxiety in surgical patients.

METHODS: A cross-cultural adaptation process using a forward/backward translation of the APAIS scale was performed. The Amharic version was then tested in 365 sampled elective adult surgical patients scheduled for surgery at Tikur Anbessa specialized Hospital. The validity of the translated version was also checked by evaluating its psychometric properties of internal validity and acceptability.

Result: The results showed that the reliability of the APAISAmharic was high (Cronbach's alpha of 0.87) and the data collected was a good fit (RMSEA of 0.04). In addition, the questionnaire was well-accepted $100 \%$ with no missing values for each dimension of the APAIS. The mean APAIS scores for total anxiety and desire for information were 11.6 and 6.0 respectively and $58.9 \%$ of the study participants had anxiety and those patients, who had some form of formal education, came from urban areas, had previous anesthesia experience and complications and who had average or high information requirement was more likely to be anxious.

CONCLUSION: APAIS-Amharic is a reliable and acceptable tool for measuring patients' preoperative anxiety and their need for information. It can be used routinely as a screening instrument at pre-anesthesia clinics to assess patients' level of anxiety.

KEYWORDS: Forward/backward translation, APAIS, preoperative anxiety, Cronbach's, alpha 


\section{INTRODUCTION}

Perioperative anxiety is a vague, uneasy feeling, the source of which is often nonspecific and unknown to the individual. It is the subjectively unpleasant feeling such as the feeling of imminent death (1). It has the potential to affect all aspects of anesthesia during preoperative, induction, post-operative and recovery periods $(3,4)$. According to Woldegerima et al, preoperative anxiety was observed in $59.6 \%$ of patients. Urban residents, low income, young age, disability, dependency, fear of death, and concern about well being of their family were found to have association with preoperative anxiety. (5)

Preoperative anxiety level is difficult to measure accurately. To measure preoperative anxiety, several validated questionnaires are currently available like Amsterdam Preoperative Anxiety Information Scale (APAIS), State-Trait Anxiety Inventory (STAI), Hospital Anxiety and Depression Scale (HADS), Visual Analogue Scale (VAS), Multiple Affect Adjective Checklist (MAACL) and newly developed specific instruments like the Anxiety Specific to Surgery Questionnaire (ASSQ) (6,7). In 1996 the Dutch group of Moerman developed the Amsterdam Preoperative Anxiety and Information Scale (APAIS) (10). This questionnaire consists of six items and is, therefore, an economical instrument. Many studies used the State-Anxiety-Scale (STAI) for evaluation of anxiety. The APAIS correlates with the STAI with $\mathrm{r}=0.74, \mathrm{r}=0.67$ as well as $\mathrm{r}=0.64$, which is a good indicator of its validity $(3,24)$. Furthermore, the APAIS was applied and proved helpful in several international studies. The APAIS has also been validated in surgical patients, whereas the STAI scale was validated in the general population (8). Unlike APAIS, the use of anxiety screening instruments like STAI usually takes a longer time. Therefore, using the APAIS that has only six items has become the standard of practice for the evaluation of perioperative anxiety in many countries. Countries like Japan, Mexico, Italy, Turkey, Thailand and South Korea have developed the
APAIS version of their language and tested its validity with excellent outcomes (3).

Ethiopia is a multi-linguistic country, and Amharic is currently used as a federal language of the country. Literature review showed that APAIS has neither been previously translated into Amharic nor used for assessment of preoperative anxiety. The primary objective of this study was to translate the APAIS into Amharic language and evaluate its validity. The secondary endpoint was to determine the prevalence of preoperative anxiety in Tikur Anbessa Specialized Hospital (TASH) and the factors associated with it.

\section{MATERIALS AND METHODS}

Design: A cross-cultural adaptation process using a forward/backward translation of the APAIS scale was performed to produce Amharic version of the APAIS scale (APAIS-Amharic). A psychometric validation was subsequently done for the adaptation of APAIS-Amharic scale (Figure 1).

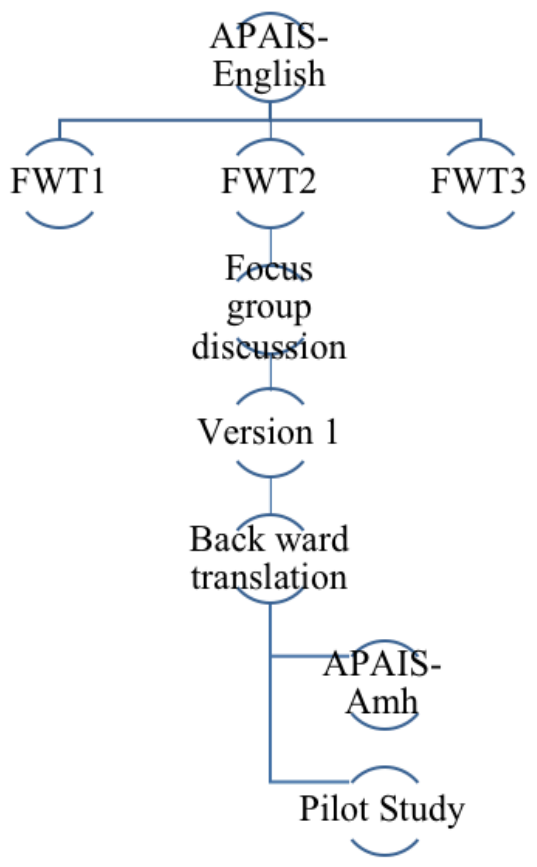

Figure 1: Process of translation and crosscultural adaptation of APAIS English to APAISAmharic 
Questionnaire of the source language: We used APAIS as a source language for translation. The questionnaire is a self-reported six-item questionnaire developed by Nelly that was used for the assessment of pre-operative anxiety. Anxiety related to anesthesia is assessed by two questions. Anxiety related to surgery and need for two also assesses information questions each. Each question is rated with a five point Likert scale (ranging from 1 'not at all' to 5 'extremely').

Process of translation and Cross-cultural adaptation: Three independent professionally trained bilingual expert translators, who are familiar with the culture of both languages were recruited. They conducted the forward translation and developed three separate Amharic versions of the questionnaire. Subsequently, in order to reconcile and synthesize the forward translation, a focus group discussion was carried out on the three versions. The focus group members were all bi-lingual and composed of three anesthesiologists, one surgeon and one English language teacher. The objective of the focus group discussion was to agree and produce a combined and culturally adapted version, which is conceptually equivalent to the original source language (Version 1). In order to resolve discordance, a blinded professional translator who had no access to the original source language did using Version 1 of the translation a backward translation. It was then compared with the original APAIS. The final version of APAISAmharic was then developed and used for our pilot study.

Pilot testing: This pilot study was done with the aim to ascertain the Amharic version of APAIS conceptual meaning correctness and the ease of administration of the tool. Ethical approval was obtained from Addis Ababa University (AAU) College of health science IRB board.

Design: Institution based prospective crosssectional study

Setting: The study was conducted in TASH, Addis Ababa, Ethiopia. TASH is Ethiopia's largest general public hospital providing a tertiary level referral treatment. It also serves as a primary teaching hospital for both clinical and preclinical disciplines. The study was conducted on elective adult surgical patients from the $1^{\text {st }}$ of July to August 30, 2019.

Study subject: The study population was adult patients of age $>18$ years admitted to the hospital for elective surgeries with ASA I-III. All the study subjects spoke and understood Amharic very well and gave consent to participate in the study. Emergency patients and patients who were not willing to give consent, patients with mental retardation, dementia, or psychiatric disorders and patients on premedication were excluded from the study.

The sample size was determined by using a single proportion for a finite population with the assumption of $95 \%$ confidence interval, $5 \%$ margin of error. A prevalence of $61 \%$ (29) was used and the calculated sample size is found to be 365. All consecutive patients that fulfill the inclusion criteria were included in the study.

Data collection and psychometric evaluation: The psychometric properties of the Amharic version were evaluated after collecting data using the translated version. Interviews with the patients were performed a night before surgery during the preoperative examination and before any premedication was given. Trained BSC nurses who were blind to the study carried out the interview. Two supervisors observed the process of data collection and also checked the consistency and cleanliness of the data daily.

The APAIS-Amharic comprises six statements. All statements were scored on a 1 to 5 Likert scale. (1-Not at all, 5- Extremely) The scores from questions 1 and 2 are added to show anesthesia-related anxiety, 4 and 5 are added together to show the patients' surgery-related anxiety while the sum of scores from questions 1, 2, 4 and 5 show the patient's total level of anxiety. In addition, scores for questions 3 and 6 are added together to identify the patent's need for information. A patient with a score of $\geq 11$ on the anxiety scale experiences anxiety. On the information scale, patients scoring 2-4 are classified as having little or no information requirements, 5-7 as having an average 
information requirement and $8-10$ as having high information requirements. Patients were also asked about the cause of their anxiety regarding the anesthesia if there were any. They could select more than one cause if it is the case.

Statistical analysis: It was made using SPSS version 24. Descriptive analysis was performed to describe the number and percentage of sociodemographic characteristics and other variables. To evaluate the correlation between variables, chi-square test of independence correlation analysis using p-value was used. The results are then presented in text, tables, and graphs based on the types of data. The psychometric evaluation of the national scaling system was done by checking its internal validity and acceptability.

Internal validity: This included checking for reliability and adequacy of the Amharic version model of the APAIS. The reliability of the internal consistency of each dimension was assessed using Cronbach's alpha coefficient. Cronbach's alpha is a function of the number of items in a test, the average covariance between item pairs and the variance of the total score. This means measuring how closely related items are as a group. For this study, Cronbach's alpha coefficient higher than 0.7 was expected for each scale.

The adequacy of the model was also explored using a global index that is responsive to sample size and complexity: the root mean square error of approximation (RMSEA) that measures how well the data collected fits the model. An RMSEA lower than 0.08 indicates a fair fit, less than 0.06 acceptable fit and less than 0.05 a good fit.

Acceptability: The percentage of missing answers was also used to explore the acceptability of the Amharic version among the patients. However, to ensure data quality, the validation analysis was not performed on records with more than $25 \%$ of the responses missing. In addition, the findings of the study were compared with other similar studies done using the state-trait anxiety inventory questionnaire. The final Amharic version of APAIS and its five point Likert scale are shown in Tables 1 and 2.

Table 2: Likert scale ranges English and Amharic version

\begin{tabular}{|c|c|c|}
\hline & English & Amharic \\
\hline 1 & Not at all & $6.8^{90}$ \\
\hline 2 & Slightly & nasmk \\
\hline 3 & Moderately & oxhhnद \\
\hline 4 & Very & nथवे \\
\hline 5 & Extremely & ᄎ. \\
\hline
\end{tabular}

Table1: Items of the Amsterdam Preoperative Anxiety and Information Scale English and Amharic version

\begin{tabular}{|c|c|c|}
\hline & Original APAIS & Translated APAIS \\
\hline 1 & I am worried about the anesthetic & 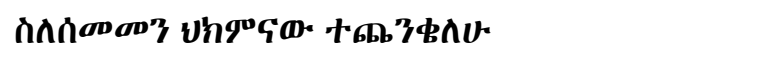 \\
\hline 2 & The anesthetic is on my mind continually & 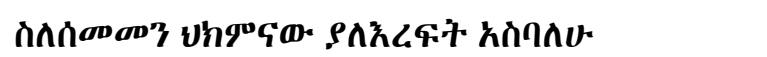 \\
\hline 3 & $\begin{array}{l}\text { I would like to know as much as possible } \\
\text { about the anesthetic }\end{array}$ & 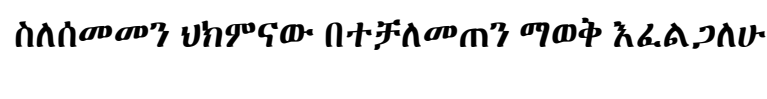 \\
\hline 4 & I am worried about the procedure & 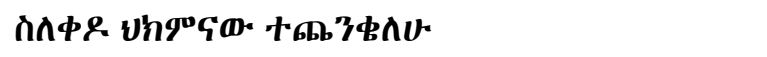 \\
\hline 5 & The procedure is on my mind continually & 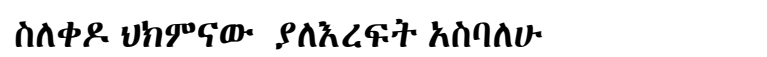 \\
\hline 6 & $\begin{array}{l}\text { I would like to know as much as possible } \\
\text { about the procedure }\end{array}$ & 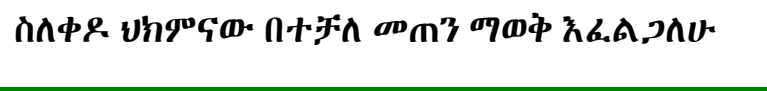 \\
\hline
\end{tabular}

\section{RESULTS}

Socio-demographic characteristics and item scores: A total of 365 patients were incorporated into this study with a $100 \%$ response rate. The study enrolled $200(54.8 \%)$ males, and $53 \%$ of the participants (194) were in the age range of 18-49. Concerning the educational status of the 
participants, 32.9\% (120) were illiterate. Out of the total sampled patients, $61.9 \%$ (226) were urban residents.

Current surgery and previous experience: Around half of the study participants were admitted for urologic $(30.7 \%)$ and general surgeries $(17.5 \%)$, followed by orthopedic $(17 \%)$, obstetrics $(9.6 \%)$ surgery, cardiothoracic (9.5\%), neurologic (7.1\%), ENT $(5.7 \%)$ and gynecologic (3.4\%) surgeries.

One-third of these patients had the experience of major surgery previously (33.7\%) while the rest did not have any previous major operation and anesthesia experience. Out of those patients who had surgery experience, the majority (66.7\%) had general anesthesia experience and $30.1 \%$ encountered surgery or anesthesia-related complications in their previous surgeries.

Out of those patients who reported the presence of either anesthesia or surgery-related complication in their previous surgeries, $40.5 \%$ had an infection as a complication followed by delayed recovery $(29.7 \%)$ and others (19\%), which included, pain and incomplete cure.

Results of the APAIS: The mean APAIS scores were as follows: anxiety for anesthesia $(5.9 \pm 2.6)$; anxiety for surgery $(5.6 \pm 2.4)$; total anxiety score $(11.6 \pm 4.6)$ and desire for information $\quad(6.0 \pm 2.4) \quad$ (Table 3$)$.

Table 3: Results of the APAIS score, mean standard deviations and Cronbach's Alpha of anxiety scores and need for information scores, in TASH, Addis Ababa, Ethiopia, July Aug 2019. (N- 365)

\begin{tabular}{lccllll}
\hline & $\mathrm{N}$ & $\begin{array}{l}\text { Min } \\
\text { Score }\end{array}$ & $\begin{array}{l}\text { Max } \\
\text { Score }\end{array}$ & Mean & SD & $\begin{array}{l}\text { Cronbach's } \\
\text { Alpha }\end{array}$ \\
\hline Anesthesia related anxiety & 365 & 2 & 10 & 5.97 & 2.65 & 0.85 \\
Surgery related anxiety & 365 & 2 & 10 & 5.60 & 2.45 & 0.84 \\
Total anxiety Score & 365 & 4 & 20 & 11.59 & 4.63 & 0.87 \\
Total need for & 365 & 4 & 20 & 6.05 & 2.37 & 0.75 \\
Information score & & & & & & \\
\hline
\end{tabular}

In addition, $58.9 \%$ of the study participants had anxiety on the APIS score, scoring 11 or more while $41.1 \%$ scored in the range of $2-11$, hence no anxiety. Around three fourth of the participants had either average $(37.5 \%)$ or high (35.9\%) information requirement regarding the anesthesia and surgery, while only $26.6 \%$ of the participants had little or no information requirement.
Out of the total interviewed 365 elective surgical patients, 312 reported at least a slight worry about the anesthesia on the first question of the APAIS. Further, these patients were asked about the reason for their worry. The unknown cause was answered by $22.4 \%$ of them, postoperative pain by $21.5 \%$, pain during surgery by $20.5 \%$ and permanent disability by $13.8 \%$ as the cause of their worry (Figure 2).

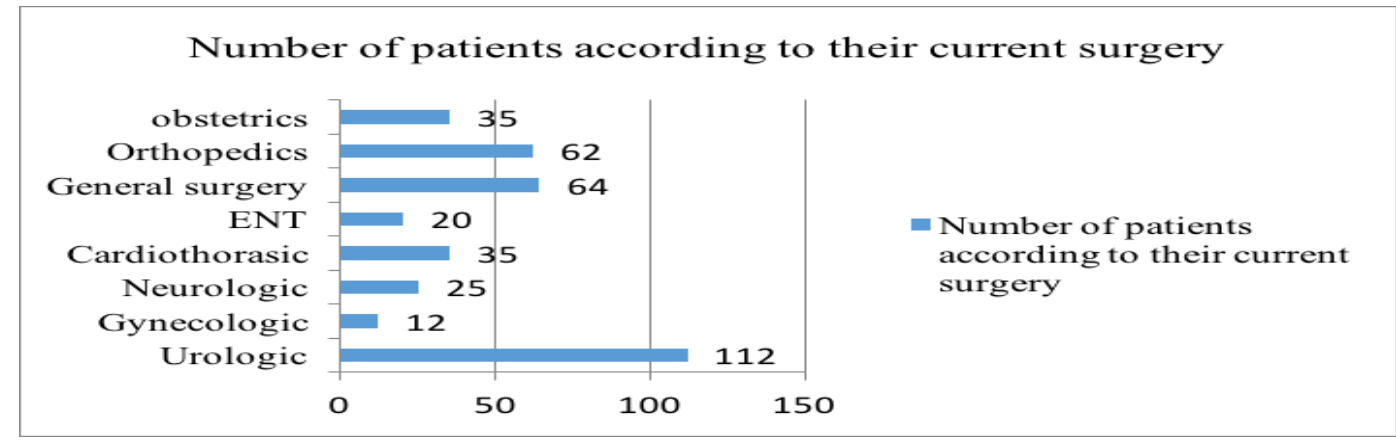

Figure 2: Causes of anesthesia related worry of elective surgical patients in TASH, Addis Ababa, Ethiopia, July-August 2019 (N-312). 
Predictors of preoperative anxiety: To evaluate the correlation between sociodemographic characteristics and anxiety score as well as the need for information and anxiety score, chi-square test of independence was performed to calculate $\mathrm{p}$-value as seen in Table 4. The proportion of patients who had anxiety (scored 11 or more on the APAIS) did not differ by age and $\operatorname{sex}\left[\mathrm{X}^{2}(1, \mathrm{~N}=365)=8.9, \mathrm{p}>.05\right]$. However, according to this study, it was found that those patients who had some form of formal education came from urban areas, had previous anesthesia experience and had previous anesthesia-related complications were more likely to be anxious $(\mathrm{P}<0.05)$.

Those who have some formal education were more likely to experience pre-operative anxiety as compared to those who do not have any education. Patients who came from urban area were more likely to be anxious than those who came from rural area. Patients with previous exposure to anesthesia who had complication were more likely to develop preoperative anxiety as compared to those who had no previous exposure and complication related to the anesthesia. In addition, while examining the relationship between patients' need for information and their anxiety score, it was found that there is a significant relationship between these variables. Those patients who had no or little information requirement regarding surgery and anesthesia were less anxious than those who had an average or high information requirement $(\mathrm{P}<0.05)$ (Table 4).

Table 4: The relationship of APAIS scores with patient characteristics and their need of Information, in TASH, Addis Ababa, Ethiopia. July-Aug 2019. (N - 365).

\begin{tabular}{llllll}
\hline & & \multicolumn{2}{c}{ Total Anxiety Score } & & \\
\cline { 3 - 4 } & & $\begin{array}{l}\text { No anxiety } \\
(2-11)\end{array}$ & $\begin{array}{l}\text { Anxiety } \\
(>=11)\end{array}$ & Total & P-value \\
\hline Age - & $18-59$ & 128 & 180 & 308 & 0.67 \\
& $>=60$ & 22 & 35 & 57 & \\
Sex & Male & 82 & 118 & 200 & 0.96 \\
& Female & 68 & 97 & 165 & \\
Educational status & No education & 62 & 58 & 120 & 0.004 \\
Residence & Some education & 88 & 157 & 245 & \\
Previous anesthesia & Urban & 79 & 147 & 226 & 0.002 \\
experience & Rural & 71 & 68 & 139 & \\
Previous Anesthesia or & No & 39 & 84 & 123 & 0.009 \\
Surgery Related & Yes & 111 & 131 & 242 & \\
Complication & No & 7 & 30 & 37 & 0.04 \\
Information requirement & No/little & 32 & 54 & 86 & \\
& Average/high & 49 & 48 & 97 & 0.03 \\
\hline
\end{tabular}

\section{Validation}

Content validity: During the focused group discussion, the panel of the five experts reviewed the translation done by the three independent professionally trained bilingual expert translators. The panel verified the conceptual equivalence of this version and

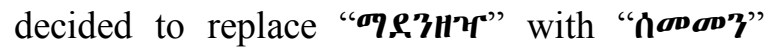
(Items 1, 2 and 3), and “中\& $\mathbf{P 7 F " ~ w i t h ~ “ \phi \& ~}$
Uh'PG" (Items 4, 5 and 6) a much more common term used by Surgical Society of Ethiopia and Ethiopian Society of Anesthesiology.

Internal validity: The internal consistency reliability of the Amharic version of the APAIS was high, i.e., the Cronbach's alpha values ranged from 0.75 to 0.85 . This shows an acceptable internal consistency of the desire for information scale and good internal consistency 
of anesthesia-related anxiety, surgical related anxiety, and global anxiety scores. In addition, the confirmatory factor analysis indicated a good fit with the root mean square error of approximation of $0.04(<.05)$.

Acceptability: From the collected data, it was found that there were no missing values for each dimension of the APAIS (100\% acceptability). These results indicate that the questionnaire was well accepted.

\section{DISCUSSION}

The APAIS has been initially designed and validated in Dutch, the construct validity was evaluated by factor analysis and external validity has also been performed. The two scales, anxiety and information need, assess important constructs for anesthesia and surgery. In this study, it was found that the reliability of the Amharic version of the APAIS was high as the internal consistency determined by the Cronbach's alpha was 0.75 for the total need for information score and 0.87 for total anxiety score (Cronbach's alpha coefficients higher than 0.7 shows acceptable internal consistency while Cronbach's alpha coefficient higher than 0.8 shows good internal consistency.) This result is similar to other studies done in French and Srilanka where Cronbach's alpha was 0.86 for the Sinhala version and ranged from 0.76 to 0.84 for the French version $(9,31)$.

In addition, the data collected fits the Amharic version of the APAIS well as it is shown by the RMSEA of 0.04 , which is a good fit $(<0.05)$. This result, when compared to the French version of the APAIS with an RMSEA result of 0.06 shows that the Amharic version has a better fit. Furthermore, the acceptability of the APAIS-Amharic was $100 \%$ as there were no missing values for each of its dimensions. Therefore, it can be used as an effective tool to measure preoperative anxiety levels in Amharic speaking patients. In this study, the overall prevalence of preoperative anxiety was $58.9 \%$ when the APAIS score was 11 and more. This result is consistent with two recent studies done in Ethiopia using the gold standard STAI scale i.e., in Gondar University Hospital (59.6\%) and
Debre Markos and Felege Hiwot hospitals $(61 \%)(5,28,32)$. This result was found to be higher than another study conducted in Nigeria where the prevalence of anxiety was $51 \%$. However, the prevalence in our study was lower than the previous study conducted in Sri Lanka using a similar tool in which the overall prevalence of preoperative anxiety was $76.7 \%$. This could be attributed to the socio-cultural differences of the two populations where our society may not have the culture to openly report about their worry regarding anesthesia and surgery out of their respect for surgeons and anesthesiologists.

In addition, the mean APAIS score for anesthesia anxiety and surgery anxiety was similar with the means of $5.9 \pm 2.6$ and $5.6 \pm 2.4$ respectively unlike the results of the study done in Pakistan where patients feared surgery significantly more than anesthesia (25). The total mean anxiety score was $11.6 \pm 4.6$, and the mean desire for information score was $6.0 \pm 2.4$ which was higher compared to the mean APAIS score for global anxiety (7.2 \pm 3.7$)$ and mean desire for information $(5.7 \pm 2.3)$ of that of the French version. This might be explained by the fact that enough time may not be given for providing preanesthesia and surgery information in our setup and hence a higher mean score of anxiety. Furthermore, out of those patients who at least reported a slight worry about the anesthesia, the top three reasons (in descending order) responsible for their anesthesia-related worry were found to be unknown cause, post-operative pain and pain during surgery. This is a different result from that of the Srilankan study where awareness during anesthesia was the number one cause for anxiety. However, the most common causes of preoperative anxiety in other studies included concern about family, fear of complication, fear of death and fear of postoperative pain $(25,26,2829)$. Therefore, for anesthesiologists, it would be important to address these causes of anesthesia-related worry of patients during their pre-anesthetic evaluation in order to alleviate their anxiety.

Different factors can affect anxiety levels in patients, which vary from country to country. In this study, the socio-demographic characteristics 
that were found to be significantly associated with preoperative anxiety were educational status and residence. However, our study showed lack of significant effect of age and gender on the level of preoperative anxiety. Those patients who had some form of formal education and came from urban areas were found to be more anxious than those who are illiterate and came from rural areas. Highly educated people who have a tendency to extrovert their feelings, information-seeking behavior, and awareness of possible complications can explain this association. Also, as they get incomplete information from different sources, their anxiety level will escalate (5). This finding is similar with a study done in Jimma, Ethiopia, (using STAI scale) and Pakistan (using VAS). The level of preoperative anxiety appeared to increase with increasing level of education and opposite to the finding of the northwest part of Ethiopia (using STAI scale) where the level of anxiety decreases with increasing level of education $(25,28,29)$. In the current study, it was also found that patients who had previous surgery or anesthesia experience and had previous anesthesia-related complications were more likely to be anxious than those who did not With the presence of previous complications, patients are more obviously become anxious because of the fear of complications happening in their current surgery as well. How ever, even in the absence of complications, patients might have experienced stressful events like the death of their neighboring patients during their last admission, which can increase their level of anxiety on their current surgery (31).

This finding is, however, different from findings of other studies that show more experienced patients were less anxious $(24,29)$. This study also showed a high positive correlation between high information seekers and anxiety scores. Those patients who had average or high information requirements were more anxious than those who had no or little information requirement regarding anesthesia and surgery. This finding is in line with other studies where patients with extremely high information requirement were anxious patients. Further, it was suggested that patients with a monitoring coping style become anxious when they are not provided with as much information as they need. However, it is also important to realize that extensive information is not always useful and may even induce anxiety particularly in patients with a blunting coping style $(10,24)$. Therefore, anxious patients may benefit from more information based on their information requirements.

Finally, although this study showed that the Amharic version of the APAIS is a reliable and acceptable tool in terms of internal validity, other domains of psychometric evaluations like differential item functioning and external validity were not done. In addition, this study did not look into the preoperative anxiety in pediatrics and emergency patients. Furthermore, the level of anxiety at pre-anesthesia clinics was not compared with that of the night before surgery, and anxious patients were not followed during and after surgery to evaluate their outcomes.

In conclusion, the Amharic version of the APAIS is a reliable and acceptable tool for measuring patients' preoperative anxiety for Amharic speaking patients. It can be used for Amharic speaking patients as a screening instrument at pre-anesthesia clinics to assess patients' levels of anxiety and hence provide a platform to clarify their doubts about anesthesia and plan for the appropriate premedications. The study also showed that the prevalence of anxiety was high and the level of anxiety was significantly associated with educational status, residence, previous surgery experience, and information requirement. Therefore, the use of the Amharic version of the APAIS for routine anxiety and the need for information assessment should be given due consideration for Amharic speaking patients. We also recommend for appropriate information to be given for patients in accordance to their need for information at pre-anesthesia clinics before surgery. Allocating enough time and providing appropriate pre-operative information for surgical patients will accordingly address their causes of worry. 


\section{ACKNOWLEDGMENT}

We acknowledge the Department of Anesthesiology for giving us the opportunity to do this research at TASH. We would like to thank Tsion Afework for her valuable comments and edition of the manuscript. We also would like to thank the data collectors and the participants of this study at large for actively participating and helping us to complete this research timely.

\section{REFERENCES}

1. Klopfenstein CE, Forster A, Van Gessel E. Anesthesia Assessment in an outpatient consultation clinic reduces preoperative anxiety. Can J Anaesth. 2000; 47(6):5115. doi: 10.1007/BF03018941.

2. Berth H, Petrowski K, Black F. The Amsterdam Preoperative Anxiety and Information scale- the first trial of a German version. psychosoc Med. 2007 Feb 20; 4: Doc 01; PMID:19742298; PMC2736533; ISSN 1860-5214

3. Buonanno P, Laiola A, Palumbo C, Spinelli G, Terminiello V, Servillo G. Italian validation of the Amsterdam preoperative anxiety and information scale. Minerva Anestesiol. 2017;83(7):705-11. doi: 10.23736/S03759393.16.11675-X. Epub 2017 Jan 17.

4. Fatma Celik and Ipek S. Edipoglu. Evaluation of preoperative anxiety and fear of anesthesia using APAIS score. Eur $J$ Med Res.2018;23:41; $\quad$ https://doi.org/ 10.1186/s40001-018-0339-4

5. Woldegerima Y.B, Fitwi G.L, Yimer H.T. and Hailekirosht. Prevalence and factors associated with preoperative anxiety among elective surgical patients at University of Gondar Hospital. Gondar, Northwest Ethiopia. International journal of surgery, 2018;10:2129. https://doi.org/10.1016/j.ijso.2017.11.001

6. Sigdel S. Perioperative anxiety: A short review. Glob Anaesthperioper Med, 2015;1(4):107-108 doi: 10.15761/GAPM.1000126

7. Karanci AN, Dirik G. Predictors of pre and post-operative anxiety in emergency surgery patients. J Psychosom Res, 2003; 55(4):363369. Doi:10.1016/s0022-3999(02)00631-1
8. Spielberger CD, Sydeman SJ. In: State Trait Anxiety Inventory and State-Trait Anger Expression Inventory, The Use of Psychological Tests for Treatment Planning and Outcome Assessment. Maruish ME, editor. Hillsdale, LEA; 1994. pp. 292321. http://dx.doi.org/10.1002/9780470479216 .corpsy0943

9. Maurice-Szamburski A, Loundou A, Capdevila X, Bruder N, Auquier P. Validation of the French version of The Amsterdam Preoperative Anxiety and Information scale (APAIS). Health Qual Life Outcomes; 2013;11:166. Doi 10.1186/1477-7525-11-166

10. Moerman N, van Dam FS, Muller MJ, Oosting H. The Amsterdam Preoperative Anxiety and Information Scale (APAIS). AnesthAnalg, 1996 Mar; 82(3):445451. Doi:10.1097/00000539-19960300000002.

11. Auquier P, Pernoud N, Bruder N, Simeoni M, Auffray J, Colavolpe C, et al. Deveopment and validation of a perioperative satisfaction questionnaire. Anesthesiology, 2005 Jun; 102(6):1116-1123. doi: 10.1097/00000542200506000-00010.

12. J C Kalkman, K Visser, J Moen, J G Bonsel, E D Grobbee, M K G Moons. Preoperative prediction of severe postoperative pain. Pain. 2003; 105(3):415-423. doi: 10.1016/S0304-3959(03)00252-5.

13. Pokharel K, Bhattarai B, Tripathi M, Khatiwada S, Subedi A. Nepalese patients' anxiety and concerns before surgery. $J$ ClinAnesth. 2011;23(5):372-8. doi: 10.1016/j.jclinane.2010.12.011.

14. Maranets I, Kain ZN. Preoperative anxiety and intraoperative anesthetic requirements. Anesth Analg. 1999;89(6):1346-51. doi:10.1097/00000539-199912000-00003.

15. Van Den Bosch JE, Moons KG, Bonsel GJ, Kalkman CJ. Does measurement of preoperative anxiety have added value for predicting postoperative nausea and vomiting? Anesth Analg, 2005;100(5):1525-32.doi: 10.1213/01.ANE.0000149325. 20542.D4.

16. Saur CD, Granger BB, Muhlbaier LH, Forman LM, McKenzie RJ, et al. Depressive symptoms and outcome of coronary artery bypass grafting. Am J Crit Care, 2001;10(1): 4-10. 
17. Scheier MF, Matthews KA, Owens JF, Schulz $\mathrm{R}$, Bridges MW, Magovern GJ et al. Optimism and re-hospitalization after coronary artery bypass graft surgery. Arch Intern Med, 1999 Apr 26; 159(8): 829-835. doi:10.1001/archinte.159.8.829

18. Baker RA, Andrew MJ, Schrader G, Knight JL. Preoperative depression and mortality in coronary artery bypass surgery: preliminary findings; ANZ J Surg,. 2001; 71(3): 139-142. doi:10.1046/j.1440-1622.2001. 02055.x

19. Perski A, Feleke E, Anderson G, Samad BA, Westerlund H, Ericsson CG et al. Emotional distress before coronary bypass grafting limits the benefits of surgery. Am Heart $J$, 1998;136(3): 510-517. doi: 10.1016/s0028703(98)70229-7.

20. Rozanski A, Blumenthal JA, Kaplan J. Impact of psychological factors on the pathogenesis of cardiovascular disease and implications for therapy, Circulation 1999; 99: 2192-2217. https://doi.org/10.1161/01.CIR.99.16.2192

21. Musselman DL, Evans DL, Nemeroff CB. The relationship of depression to cardiovascular disease: epidemiology, biology, and treatment; Arch Gen Psychiatry, 1998;55(7): 580-592. doi:10.1001/archpsyc.55.7.580

22. Kubzansky LD, Kawachi I, Weiss ST, Sparrow D. Anxiety and coronary heart disease: a synthesis of epidemiological, psychological, and experimental evidence. Ann Behav Med Spring, 1998; 20(2): 47-58. doi: 10.1007/BF0288448.

23. Perks A, Chakravarti S, Manninen P. Preoperative anxiety in neurosurgical patients. $J$ Neurosurg Anesthesiol, 2009;21(2):127-30 doi:10.1097/ANA.0b013e31819a6ca3

24. Nishimori M, Moerman N, Fukuhara S, van Dam FS, Muller MJ, Hanaoka $\mathrm{K}$, et al. Translation and validation of the Amsterdam preoperative anxiety and information scale(APAIS) for use in Japan. Qual Life Res 2002;11(4):361-4. doi:10.1023/a:1015561129899

25. Haque SN, Ara A, Imtiaz A, Hamirani M. Validity of visual analogue scale in assessment of Anxiety: a comparative study in Anesthesia clinic. Annals of Abbasi Shaheed Hospital and Karachi Medical Dental College, 2004; 9: 571-574

26. AkinsuloreA, Owojuyigbe AM, Faponle AF, Fatoye FO. Assessment of preoperative and postoperative anxiety among elective major surgery patients in a tertiary hospital in Nigeria. Middle East J Anesthesiol. 2015; 23(2): 235-40.

27. Kamau A, Munng'ayi V, Yonga G. The effect of a preanaesthesia clinic consultation on adult patient anxiety at a tertiary hospital in Kenya: a cohort study. Afri Health Sci. 2017;17(1): 138-147.

https://dx.doi.org/10.4314/ahs.v17i1.18

28. Nigussie S, Belachew T, Wolancho W. Predictors of preoperative anxiety among surgical patients in Jimma University specialized teaching hospital, South Western Ethiopia. BMC Surg. 2014; 14:67. $\mathrm{http}: / /$ www.biomedcentral.com/1471$2482 / 14 / 67$

29. Henok M, Mulatu A, Mezinew S, Getenet D, and Tesfu Z. Preoperative anxiety and associated factors among adult surgical patients in Debre Markos and Felege Hiwot referral hospitals, Northwest Ethiopia. BMC Anesthesiology; 2018; 18:155. https://doi.org/10.1186/s12871-018-0619-0

30. Anne Thushara Matthias, Dharmanbandhu Nandadeva Samarasekera. Preoperative anxiety in surgical patients - experience of a single unit. Acta Anaesthesiologica Taiwanica; 2012;50(1): 3-6. https://doi.org/10.1016/j.aat.2012.004

31. Aalouane R, Rammouz I, Tahiri-Alaoui D, Elrhazi K, Boujraf S. Determining factors of anxiety in patients at the preoperative stage; Neurosciences (Riyadh), 2011;16(2):146-9.

32. Abdulaziz B, Laurence B, Neil D. The Amsterdam preoperative anxiety and information scale provides a simple and reliable measure of preoperative anxiety. Canadian Journal of anesthesia, 2002; 49 (8): 792-8. DOI : 10.1007/BF03017410 